\title{
Modificações vocais acústicas produzidas pela fonação reversa
}

\section{Acoustic vocal modifications produced by reverse phonation}

\author{
Leila Susana Finger ${ }^{1}$, Carla Aparecida Cielo ${ }^{2}$
}

\begin{abstract}
RESUMO
Objetivo: Descrever as modificações vocais acústicas e as sensações ocorridas após a técnica vocal de fonação reversa em mulheres adultas jovens, sem queixas vocais e com laringe normal. Métodos: Trinta e duas mulheres adultas jovens submeteram-se à avaliação otorrinolaringológica e triagem fonoaudiológica para descartar possíveis alterações que pudessem interferir nos resultados da pesquisa; tiveram amostras vocais coletadas antes e após realizarem três séries de 15 repetições de fonação reversa, em tempo máximo de fonação com tom e intensidade habituais, e 30 segundos de repouso passivo entre cada série. Após, responderam a um questionário referente às sensações percebidas. A análise vocal acústica foi realizada através do software Praat (versão 4.6.10) e os dados analisados por meio da estatística descritiva e pelo teste de Wilcoxon, com nível de significância de 5\%. Resultados: Aumento estatisticamente significativo da frequência fundamental e da frequência máxima; diminuição da frequência mínima; aumento das medidas de Jitter, exceto da medida de Jitter local-absoluto que diminuiu; diminuição das medidas de Shimmer, relação ruído/harmônico (NHR) e relação harmônico/ruído (HNR); e predomínio das sensações positivas. Conclusão: A fonação reversa pareceu promover efeito positivo sobre a vibração da mucosa das pregas vocais e sobre o seu alongamento. Sugere efeito sobre a musculatura, favorecendo mudanças de frequência fundamental; e sobre sua homogeneização e modificação da camada de muco. Além disso, promoveu melhora global do sinal vocal e das sensações durante sua produção.
\end{abstract}

Descritores: Fonação; Voz; Treinamento da voz; Qualidade da voz; Distúrbios da voz

\section{INTRODUÇÃO}

A fonação reversa, inicialmente descrita por meio da análise radiológica da laringe ${ }^{(1)}$, é a produção vocal que ocorre durante a inspiração. Acontece espontaneamente em diversas situações, como no riso, no choro e no suspiro ${ }^{(2)}$. Surge da pressão gerada acima do nível da glote, através da entrada turbulenta de ar, aduzindo as verdadeiras pregas vocais. Durante essa manobra, ocorre o relaxamento dos ventrículos, a ampliação do vestíbulo laríngico e a adução glótica em toda a extensão $0^{(3-7)}$, alongando as pregas vocais ${ }^{(4,7)}$.

$\mathrm{Na}$ literatura, encontram-se relatos descrevendo um significativo aumento da perturbação e instabilidade do contato entre as pregas vocais durante a fonação reversa se comparada à fonação normal ${ }^{(6,8)}$. Também são referidos o afastamento das pregas ventriculares, a queda da pressão subglótica (causando distensão dos ventrículos), e a visualização mais definida dos seios piriformes ${ }^{(1-7)}$.

Trabalho realizado no Programa de Pós-graduação em Distúrbios da Comunicação Humana da Universidade Federal de Santa Maria - UFSM - Santa Maria (RS), Brasil.

(1) Mestre, Fonoaudióloga clínica - São Leopoldo (RS), Brasil.

(2) Doutora, Professora Adjunto do Departamento de Fonoaudiologia da Universidade Federal de Santa Maria - UFSM - Santa Maria (RS), Brasil. Endereço para correspondência: Leila Susana Finger. R. João Neves da Fontoura, 716/404, Centro, São Leopoldo - RS, CEP 93010-050. E-mail: leilafiner@yahoo.com.br

Recebido em: 7/2/2008; Aceito em: 29/7/2008
Além de estar presente no choro dos neonatos ${ }^{(2)}$, a fonação reversa é utilizada na prática otorrinolaringológica, auxiliando a detecção de lesões de massa de prega vocal durante o exame de laringoscopia ${ }^{(3,7)}$. É utilizada, ainda, na prática clínica fonoaudiológica, como técnica de reabilitação $0^{(5,7-8)}$, podendo, inclusive, mostrar-se eficaz na melhora da fluência de indivíduos com gagueira $^{(7)}$ e de alguns portadores de disfonia espasmódica ${ }^{(4,7-8)}$.

A técnica da fonação reversa é utilizada na reabilitação vocal em casos de afonia funcional psicogênica, puberfonias, fendas triangulares médio-posteriores, fendas por paresias e paralisias das pregas vocais, fonação com pregas vestibulares, e fonação ariepiglótica ${ }^{(9)}$. Diversos autores indicam o uso desta técnica na intervenção de disfonias espasmódicas, como fonação alternativa, não exigindo o resgate da fonação fisiológica, ou seja, da fonação expiratória ${ }^{(4-5,9)}$.

Quanto à terapia, observou-se que a fonação reversa é utilizada nas disfonias psicogênicas pela mudança imediata do ajuste muscular e nas alterações de muda vocal, pois a emissão que se segue à fonação reversa ocorre, geralmente, em registro modal grave. Nos casos de fendas glóticas e paralisia de pregas vocais, é utilizada por promover a adução das pregas vocais ${ }^{(5,9)}$. Nos casos de fonação ariepliglótica ou fonação ventricular, a fonação reversa restabelece a vibração das pregas vocais, devendo ter seu uso reduzido gradualmente até a recuperação da fonação ${ }^{(9)}$. Na intervenção em casos de gagueira, a manobra de Valsava, fechamento apertado das 
pregas vocais, não ocorre durante inspiração, favorecendo-se a fluência por meio da fonação reversa ${ }^{(7)}$.

De forma geral, no que diz respeito às características acústicas, foram encontrados na literatura registros de que a fonação reversa promove o aumento da frequência fundamental ${ }^{(5-8)}$, a manutenção dos níveis de intensidade ${ }^{(4,7-8)}$, aumento das medidas de Jitter $^{(6)}$. Acredita-se que estas medidas reflitam um impacto positivo quanto à utilização da técnica, uma vez que, parecem demonstrar um aumento da mobilização, a favor da gravidade, da mucosa da prega vocal. Não foram encontrados registros sobre medidas de ruído, Shimmer, frequência máxima e frequência mínima, após a produção da fonação reversa.

Na literatura mundial, sobretudo na nacional, são poucos os relatos de estudos que descrevem as modificações vocais decorrentes das técnicas vocais, em especial, pesquisas que propiciem maior entendimento das modificações vocais produzidas pela fonação reversa ${ }^{(8)}$.

Neste sentido, situa-se a relevância do presente trabalho, uma vez que pretende contribuir com a melhor compreensão das modificações acústicas produzidas pela fonação reversa em laringes normais em função de sua correlação com a fisiologia laríngea sem afecções. Este maior conhecimento dos efeitos da fonação reversa na normalidade pode subsidiar a implementação de novas pesquisas, que avaliem objetivamente os efeitos da fonação reversa em grupos com diferentes afecções vocais e laríngeas, permitindo parâmetros de comparação.

Além disso, os resultados da fonação reversa encontrados em laringes normais podem amparar a técnica como um exercício a ser utilizado em aquecimento vocal naqueles sujeitos que não apresentam problemas laríngeos.

O presente estudo visa a descrever as modificações vocais acústicas ocorridas após a produção da técnica vocal de fonação reversa em mulheres adultas sem queixas vocais e com laringe normal; averiguar as sensações percebidas pelos sujeitos após a produção da técnica; e verificar a existência de possíveis correlações entre os tipos de modificações vocais encontradas.

\section{MÉTODOS}

A pesquisa caracteriza-se por ser uma investigação de campo, exploratória, de cunho quantitativo e qualitativo, cujo projeto foi aprovado pelo Comitê de Ética em Pesquisa da instituição de origem, sob o número 024/2006. A populaçãoalvo recebeu os esclarecimentos necessários sobre o estudo e foi convidada a ler e assinar o Termo de Consentimento Livre e Esclarecido (TCLE), como recomenda a norma 196/96 da Comissão Nacional de Ética em Pesquisa - CONEP/1996.

Os critérios de inclusão dos sujeitos na pesquisa foram: adesão ao TCLE; gênero feminino; idades entre 18 e 40 anos, pois se acredita que nessa faixa etária o aparelho fonador ainda não sofreu a influência das alterações hormonais e estruturais do envelhecimento ${ }^{(10)}$, como também não sofre mais as alterações do período da muda vocal, que na mulher ocorre entre os 12 e 14 anos $^{(11)}$; fechamento glótico completo ou presença de fenda posterior grau I, pois representa o padrão laríngeo feminino e não apresenta impacto sobre a fonação ${ }^{(9,12)}$.

Os critérios de exclusão foram: queixas vocais, como rouquidão, fadiga vocal, falhas na voz ou ardência na garganta, visto que estes são sintomas sugestivos de algum tipo de alteração vocal orgânica ou comportamental ${ }^{(5,9,12)}$, podendo interferir nos resultados da pesquisa; patologias laríngeas, pois distúrbios no nível laríngeo poderiam comprometer os resultados da avaliação ${ }^{(5,9,12)}$; apresentar história pregressa de doenças neurológicas, endocrinológicas, psiquiátricas ${ }^{(9,12)} \mathrm{ou}$ gástricas $^{(13)}$, que poderiam influenciar na performance vocal ou no entendimento das ordens durante as avaliações ${ }^{(5,12)}$; alterações hormonais decorrentes de gravidez ou período menstrual e pré-menstrual, coletadas através de anamnese aberta $^{(14)}$; estar com gripe e/ou alergias respiratórias ${ }^{(15)}$, porque ambos podem causar edema nas pregas vocais, ou outra doença que pudesse limitar o desempenho na execução da técnica de fonação reversa, no dia das avaliações; hábitos de etilismo e tabagismo ${ }^{(5,9,12)}$, já que esses agentes são agressivos à laringe e podem originar problemas vocais orgânicos; ter realizado tratamento fonoaudiológico e/ou otorrinolaringológico prévios, para descartar a possibilidade de que o sujeito tivesse qualquer patologia vocal (mesmo já tratada) ou um condicionamento vocal através de treinamento com técnicas vocais; conhecimento da técnica vocal estudada; alterações auditivas, pois elas podem modificar o automonitoramento da voz, comprometendo a qualidade vocal ${ }^{(9,12)}$; alterações do sistema estomatognático que pudessem interferir na articulação da fala, comprometendo a voz ${ }^{(12)}$; não habilidade de realização da técnica de fonação reversa com sucesso; cantar em coros, a fim de evitar que o sujeito já possuísse noções de técnicas vocais ou tivesse sua voz "trabalhada".

Dessa forma, foram verificadas as modificações vocais, ocorridas após a produção da fonação reversa, em 32 mulheres, com a média de 20,56 anos, voluntárias, adultas, brasileiras, sem queixas vocais e/ou presença de patologias laríngeas.

Após a aplicação do TCLE, que garante os aspectos bioéticos da pesquisa, foram realizadas algumas avaliações com o objetivo de descartar possíveis alterações que pudessem interferir nos resultados da pesquisa, conforme os critérios de inclusão e de exclusão dos sujeitos. Inicialmente, os participantes responderam a um questionário. Depois, foi realizada uma avaliação otorrinolaringológica, por meio de laringoscopia indireta, com o objetivo de descartar patologias laríngeas. Foram realizados ainda, exame orofacial e avaliação audiométrica, por meio de varredura de tons puros nas frequências de 250 , $500,1000,2000,4000,6000$ e $8000 \mathrm{~Hz}$, a $25 \mathrm{~dB}$, somente pela via aérea, em cabine acusticamente tratada, com audiômetro modelo Fonix FA 12 Digital.

Os voluntários que apresentaram alteração em alguma das avaliações foram descartados da pesquisa e encaminhados para avaliações mais completas. Aqueles que se encaixaram nos critérios de inclusão iniciaram a coleta de dados. Inicialmente, foi colhida a emissão sustentada da vogal /a/, pedindo-se ao sujeito que ficasse em pé, com os braços estendidos ao longo do corpo. O microfone, acoplado ao gravador digital da marca Creative Labs, modelo MuVo Tx FM, foi posicionado em ângulo de $90^{\circ}$ graus da boca do sujeito, mantendo-se a distância de $4 \mathrm{~cm}$ entre o microfone e a boca ${ }^{(9,16-18)}$. Foi solicitada a realização da emissão sustentada, em frequência e intensidade habituais, após inspiração profunda, emitindo o som em tempo máximo 
de fonação (TMF), sem uso de ar de reserva expiratória.

Por se tratar de indivíduos sem treinamento vocal, optouse pela realização das emissões em TMF, inclusive durante a realização da técnica. A emissão em TMF respeita a variação individual da capacidade vital e da coordenação pneumofonoarticulatória, prevenindo a fadiga vocal.

Em um segundo momento, os participantes foram orientados a produzir três séries, com 15 repetições cada $^{(19)}$, de fonação reversa com inspirações nasais. Foram orientados a esvaziar os pulmões e realizar a emissão "ihn" durante a inspiração nasal ${ }^{(8)}$. Todas as produções foram acompanhadas, realizando-se as correções necessárias para que todos os indivíduos realizassem a técnica corretamente e de forma similar.

A técnica foi executada com o indivíduo sentado de forma confortável, sem deslocamento cervical, com os pés apoiados no chão, coluna ereta, mantendo o ritmo constante entre um exercício e outro. Após cada série de 15 repetições, houve um repouso passivo de 30 segundos ${ }^{(19)}$, durante o qual os sujeitos permaneceram em silêncio absoluto. Durante a realização da técnica, os indivíduos puderam ingerir água, em função do elevado aumento de fluxo aéreo naso-faríngeo que ocorre durante a execução da fonação reversa nasal. A ingestão de água não foi considerada uma variável interveniente nos resultados devido ao fato de ela não penetrar diretamente na laringe.

Após a realização das séries da técnica, os participantes tiveram a vogal /a/ colhida nas mesmas condições pré-técnica e responderam a um questionário fechado referente às sensações desagradáveis percebidas, como tontura, desconforto laríngeo, diminuição do TMF (tempo mais "curto" ou mais "comprido" do /a/), dentre outras. Também relataram as sensações positivas decorrentes da execução da técnica, como voz mais solta, sendo que a sensação de pigarro foi considerada positiva, em função de evidenciar a liberação do muco que recobre a mucosa do trato vocal.

Esta foi considerada uma sensação positiva, embora se saiba que o pigarro também está relacionado ao excesso de esforço e tensão na realização de técnicas vocais, e, nesta pesquisa, também ao possível ressecamento causado pela fonação reversa. Como as sensações de esforço, cansaço, ressecamento, e tensão foram pouco referidas pelos sujeitos, após a realização da fonação reversa, considerou-se que o pigarro, neste contexto, sugere melhora da lubrificação e, portanto, sensação positiva.

Para a análise acústica da voz, foram extraídos os 3,5 segundos iniciais da emissão da vogal /a/, sendo excluído o início da emissão para que o ataque vocal não interferisse na análise dos dados ${ }^{(20)}$

As medidas, obtidas através do software Praat (versão 4.6.10) $)^{(21)}$, utilizado em diversos estudos ${ }^{(16,17,22,23,24)}$, foram: frequência fundamental $\left(\mathrm{f}_{0}\right)$; frequência mínima; frequência máxima; Jitter (local); Jitter (local, absoluto); Jitter (rap); Jitter (ppq5); Jitter (ddp); Shimmer (local); Shimmer (local, dB); Shimmer (apq3); Shimmer (apq5); Shimmer (apq11); Shimmer (dda); relação ruído/harmônico (NHR); relação harmônico/ruído (HNR). Tais medidas englobam todas as oferecidas pelo programa, sendo importantes na análise pelo fato de fornecerem subsídios sobre os níveis de aperiodicidade, de estabilidade, de ruído, e de frequência do sinal vocal.
Os valores de $150 \mathrm{a} 250 \mathrm{~Hz}$ para $\mathrm{f}_{0}^{(25)}$ foram considerados neste estudo, por englobarem os resultados de normalidade encontrados por estudos posteriores ${ }^{(26-27)}$.

Em algumas das demais medidas os autores do programa ${ }^{(21)}$ propõem uma analogia aos valores considerados normais pelo programa Multi-Dimensional Voice (MDVP) da Kay Elemetrics ${ }^{\circledR}$ (Tabela 1).

Tabela 1. Analogia entre valores de normalidade Praat x MDVP para vozes femininas

\begin{tabular}{lccc}
\hline Medida Praat & $\begin{array}{c}\text { Medida MDVP } \\
\text { equivalente }\end{array}$ & $\begin{array}{c}\text { Normalidade } \\
\text { MDVP } \\
\text { (mulheres) }\end{array}$ & $\begin{array}{c}\text { Tresh } \\
\text { MDVP }\end{array}$ \\
\hline Jitter local (\%) & Jitt & & $<1,040$ \\
Jitter local, absoluto & Jitta & $<83,200$ \\
( $\mu$ s) & Jitter rap & & $<0,680$ \\
Jitter rap (\%) & Jitter (PPQ) & 0,36 & $<0,840$ \\
Jitter ppq5 (\%) & Original do Praat & - & - \\
Jitter ddp (\%) & Shim & $<3,810$ \\
Shimmer local (\%) & ShdB & - & - \\
Shimmer local dB & Original do Praat & - & - \\
(dB) & Original do Praat & - & $<350$ \\
Shimmer apq3 (\%) & APQ & 1,39 & $<3,070$ \\
Shimmer apq5 (\%) & - & - \\
Shimmer apq11 (\%) & Original do Praat & - & $<0,190$ \\
Shimmer dda (\%) & NHR & 0,11 & - \\
NHR & Original do Praat & - & \\
HNR (dB) & & &
\end{tabular}

Os dados foram analisados estatisticamente, por meio da estatística descritiva, e foi aplicado o teste não paramétrico de Wilcoxon, utilizado para comparar as produções pré e pósfonação reversa, a um nível de significância de 5\%.

\section{RESULTADOS}

Apresentaram-se como voluntários 48 sujeitos, dos quais 16 não passaram pelos critérios de inclusão e de exclusão da pesquisa. Foram excluídos: cinco indivíduos na aplicação do questionário, dois por serem fumantes e três por terem distúrbios alérgicos; oito por não terem disponibilidade em realizar a avaliação ORL; um por ter mais de 40 anos; dois por serem menores de 18 anos. Encaixaram-se nos critérios de inclusão 32 indivíduos, voluntários, do sexo feminino, com idades entre 18 e 39 anos e média de 20,56 anos.

Os resultados da avaliação acústica vocal, ilustrados na Tabela 2, mostraram aumento estatisticamente significativo da $\mathrm{f}_{0}$ (aumento médio de $8,65 \mathrm{~Hz}$ ) e da frequência máxima (aumento médio de 13,51 Hz).

Observou-se aumento das medidas de Jitter (local) (média de $0,018 \%$ ), de Jitter (rap) (média de 0,008\%), de Jitter (ppq5) (média de 0,017\%), e de Jitter (ddp) (média de 0,052\%).

Constatou-se diminuição, embora não estatisticamente significativa das medidas de frequência mínima (média de 0,84Hz), de Jitter (local-absoluto) (média de 0,173 ( $\mu \mathrm{s})$ ), de 
Tabela 2. Resultado da análise acústica, por meio do software Praat, pré e pós-fonação reversa

\begin{tabular}{|c|c|c|c|c|c|c|}
\hline Variável & Pré (Média) & Desvio-padrão & Pós (Média) & Desvio-padrão & Valor de $p$ & $\begin{array}{c}\text { Variação (Média) } \\
\text { pré e pós }\end{array}$ \\
\hline $\mathrm{f}_{0}(\mathrm{~Hz})$ & 213,05 & 3,86 & 221,64 & 25,74 & $0,0301^{*}$ & $+8,59$ \\
\hline Frequência mínima $(\mathrm{Hz})$ & 196,86 & 35.53 & 196,02 & 49,25 & 0,3577 & $-0,84$ \\
\hline Frequência máxima $(\mathrm{Hz})$ & 216,28 & 21,25 & 229,79 & 25,74 & $0,0068^{*}$ & $+13,51$ \\
\hline Jitter local (\%) & 0,470 & 0,139 & 0,488 & 0,159 & 0,8194 & $+0,018$ \\
\hline Jitter local, absoluto ( $\mu \mathrm{s})$ & 22,288 & 6.909 & 22,115 & 6,710 & 0,9038 & $-0,173$ \\
\hline Jitter rap (\%) & 0,282 & 0,088 & 0,290 & 0,100 & 0,8561 & $+0,008$ \\
\hline Jitter ppq5 (\%) & 0,269 & 0,088 & 0,286 & 0,090 & 0,6288 & $+0,017$ \\
\hline Jitter ddp (\%) & 0,820 & 0,291 & 0,872 & 0,300 & 0,7574 & $+0,052$ \\
\hline Shimmer local (\%) & 3,718 & 1,710 & 3,710 & 1,823 & 0,5502 & $-0,08$ \\
\hline Shimmer local dB (dB) & 0,290 & 0,156 & 0,285 & 1,823 & 0,5682 & $-0,005$ \\
\hline Shimmer apq3 (\%) & 2,230 & 0,852 & 2,224 & 0,891 & 0,7677 & $-0,006$ \\
\hline Shimmer apq5 (\%) & 2,711 & 1,160 & 2,640 & 1,300 & 0,5866 & $-0,071$ \\
\hline Shimmer apq11 (\%) & 2,883 & 1,738 & 2,698 & 1,938 & 0,3043 & $-0,185$ \\
\hline Shimmer dda (\%) & 5,783 & 2,556 & 5,756 & 2,665 & 0,7321 & $-0,027$ \\
\hline $\mathrm{NHR}$ & 0,035 & 0,042 & 0,025 & 0,011 & 0,4051 & $-0,01$ \\
\hline HNR (dB) & 16,767 & 1,970 & 17,116 & 1,936 & 0,4481 & $+0,349$ \\
\hline
\end{tabular}

Legenda: * valores significantes

Teste de Wilcoxon

Shimmer (local) (média de 0,08\%), de Shimmer (local, dB) (média de -0,005 dB), de Shimmer (apq3) (média de -0,006\%), de Shimmer (apq5) (média de -0,071\%), de Shimmer (apq11) (média de -0,185\%), de Shimmer (dda) (média de -0,185\%), da relação ruído/harmônico (NHR) (média de -0,01), e da relação harmônico/ruído (HNR) (média de -0,349).

No que diz respeito às sensações proprioceptivas, apresentadas na Figura 1,31 sujeitos (96,88\%) referiram voz mais solta e melhora para falar; 29 (90,63\%), melhor projeção da voz; sete $(21,88 \%)$, sensação de secreção na garganta; seis $(18,75 \%)$, vontade de pigarrear; dois $(6,25 \%)$, sensação desagradável, ressecamento; e um $(3,13 \%)$ redução do TMF (Tabela 2$)$.

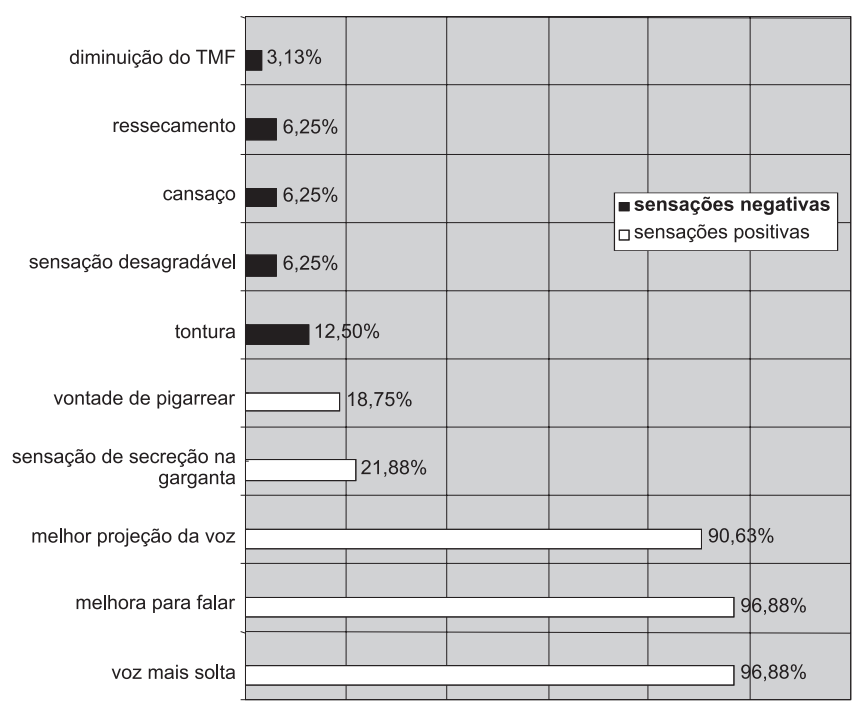

Figura 1. Sensações percebidas pelos sujeitos após a realização da fonação reversa

\section{DISCUSSÃo}

Durante a manobra da fonação reversa, ocorre a adução glótica em toda a extensão ${ }^{(3-6)}$, gerando um alongamento das pregas vocais ${ }^{(4,7-8)}$.

Sabe-se que características como o comprimento, a massa e a tensão das pregas vocais, durante a vibração, estão envolvidas na modificação da frequência da voz. Quanto mais as pregas vocais forem alongadas, mais rápido se realizarão os ciclos glóticos, e mais aguda será a frequência produzida ${ }^{(5,9,12)}$.

No presente estudo, observou-se aumento estatisticamente significativo da $\mathrm{f}_{0}$, se comparadas as emissões pré e pós-fonação reversa (Tabela 2), o que vai ao encontro dos resultados de diversos estudos que descrevem aumento significativo da $\mathrm{f}_{0}$ durante a fonação reversa, se comparada à fonação expiratória ${ }^{(2,5-8,28)}$.

Em estudo realizado com mulheres ${ }^{(8)}$, foi observado aumento estatisticamente significativo da $\mathrm{f}_{0}$ durante a fonação reversa (média de 285,833 Hz), quando comparada às produções em fonação expiratória (média de 234,467 Hz), um aumento médio de 51,366 Hz. Resultado similar ao encontrado por outro estudo ${ }^{(28)}$ realizado com 15 homens e 15 mulheres, com média de idade de 26 anos, sobre as características acústicas das vogais /i/, /u/ e /a/, no qual foi observado um aumento médio de 60 $\mathrm{Hz}$ durante a produção em fonação reversa, quando comparada à produção em fonação habitual.

Na respiração confortável, durante o sono, a ação do CT (músculo cricotireóideo) é predominantemente inspiratória. No estado de consciência, geralmente está ausente durante a inspiração, mas pode ser recrutada em caso de oclusão de vias aéreas ou por inspiração voluntária profunda ${ }^{(12,29)}$, como ocorre durante a fonação reversa.

Acredita-se que a atividade do CT, ativado durante a inspiração, possa contribuir para o aumento da tensão das pregas 
vocais durante a fonação reversa e, consequentemente, para o aumento da $\mathrm{f}_{0}$ e do pitch $^{(8)}$. Esse ajuste propicia também, o alongamento do músculo tireoaritenódeo (TA), músculo de contração rápida e altamente fatigável; fadiga que gera queda da resistência fonatória ${ }^{(12)}$. Acredita-se que a fonação reversa contribua com a diminuição da tensão muscular dos feixes do TA, afastando as pregas vestibulares e deixando a mucosa mais solta para vibrar.

Esse ajuste, além de auxiliar o aumento da $\mathrm{f}_{0}$ parece estar estreitamente relacionado com o aumento estatisticamente significativo da frequência máxima e também com a diminuição, embora não significativa estatisticamente, da frequência mínima nas produções pós-fonação reversa deste estudo (Tabela 2). Pôde-se constatar, ainda, a ocorrência de aumento da extensão fonatória do grupo, ou seja, diminuição da frequência mínima e aumento da frequência máxima, após a realização da fonação reversa, o que sugere a possibilidade de utilização da fonação reversa junto ao canto profissional.

Quanto ao aumento do desvio-padrão das medidas de $\mathrm{f}_{0}$, frequência máxima, e frequência mínima, supõe-se a influência da resposta individual de cada sujeito frente às modificações dos ajustes motores e teciduais da mucosa após a realização da fonação reversa. Os sujeitos apresentaram maior variabilidade do desvio-padrão na $\mathrm{f}_{0}$ e na frequência mínima, mostrando que as respostas são muito individuais, mas que, em relação ao desvio-padrão, do mesmo modo que ao se analisar a variação da $\mathrm{f}_{0}$ e da frequência máxima, infere-se a forte influência da fonação reversa sobre o alongamento das pregas vocais, pois o desvio-padrão aumentou principalmente na a $\mathrm{f}_{0}$ e na frequência máxima, sugerindo maior flexibilidade muscular que propicia maior possibilidade de variação individual na produção de frequências, ocasionando aumento do desvio-padrão dessas medidas.

Considera-se que o aumento das medidas de Jitter (local), de Jitter (rap), de Jitter (ppq5), de Jitter (ddp), e a diminuição do Jitter (local-absoluto), embora não estatisticamente significativos (Tabela 2), também estejam relacionados a esse ajuste, visto que os resultados de todas essas medidas parecem mostrar que o período médio dos ciclos das emissões analisadas diminuiu, ou seja, houve mais ciclos por segundo no pós-técnica, confirmando os resultados de aumento da $\mathrm{f}_{0} \mathrm{e}$ da frequência máxima.

Acredita-se que o aumento das medidas de Jitter após a fonação reversa, compatível com outro estudo(6), esteja relacionado ao aumento da mobilização da mucosa e seja um resquício dessa grande mobilização a favor da gravidade ao voltar à emissão em registro modal. É possível que após certo tempo, os sujeitos resgatem a coordenação pneumofonoarticulatória habitual, não apresentando aumento das medidas de Jitter, porém a confirmação desta hipótese demanda outras pesquisas.

O conhecimento sobre a atividade da musculatura laríngea envolvida no aumento da $\mathrm{f}_{0}$ associado a estudos sobre as modificações laríngeas durante a fonação reversa, pode auxiliar o profissional fonoaudiólogo a tomar decisões mais adequadas ao utilizá-la como técnica de tratamento ${ }^{(8)}$.

$\mathrm{Na}$ fonação reversa, o som provém da vibração ampla e sincrônica da mucosa, em sentido inverso ao da fonação expira- tória, e da força pró-gravidade, decorrente do aumento da pressão supraglótica, que favorece a entrada de ar e a mobilização mucosa, possibilitando a ocorrência do efeito de Bernoulli ${ }^{(8,30)}$. São mobilizadas as estruturas supraglóticas, ocorrendo diminuição da constrição mediana e ântero-posterior ${ }^{(1)}$.

A manobra inspiratória auxilia a regular a abertura glótica e a taxa de ar inspiratório, que determinam a queda da pressão transglótica associada ao efeito de Bernoulli. Durante essa manobra, a face superior das pregas vocais interage com a queda da pressão transglótica e, provavelmente, a pressão traqueal negativa cause uma mudança na força vetorial, ou seja, o vetor soma das forças direcionais gerando deslocamento dos tecidos a favor da gravidade ${ }^{(8,30)}$.

Essa vibração da mucosa a favor da gravidade promove a sua soltura e mobilização, propiciando o deslizamento da cobertura mucosa das pregas vocais sobre seu corpo, melhorando a circulação sanguínea periférica e, ainda, massageando a mucosa que as reveste ${ }^{(5)}$.

Ao ser efetiva na mobilização da mucosa das pregas vocais, a técnica contribui com a melhora da qualidade vocal e a redução dos ruídos associados à emissão fônica ${ }^{(12)}$, pois propicia o massageamento da mucosa que promove a sua homogeneização interna e a remoção do muco que recobre o epitélio das pregas vocais. Os resultados do presente estudo corroboram essas afirmações, uma vez que ocorreu diminuição, embora não estatisticamente significativa, das medidas de ruído NHR e HNR e das medidas de Shimmer (Tabela 2).

No NHR, a dimuniuição da média, a variação negativa, e a diminuição do desvio-padrão mostram, respectivamente, que houve diminuição do ruído à emissão (média e variação) e maior estabilidade desta diminuição no pós-técnica, pois o desvio-padrão diminuiu.

Em um estudo ${ }^{(6)}$ foi encontrado aumento significativo da frequência de perturbação e maior instabilidade do grau de contato das pregas vocais durante a fonação reversa. O que vai ao encontro dos resultados obtidos referentes ao aumento das medidas de Jitter, embora não estatisticamente significativas (Tabela 2), na produção pós-fonação reversa.

As medidas acústicas de perturbação podem ser consideradas correlatos acústicos da instabilidade do sinal, resultantes da redução do controle sobre o sistema fonatório ${ }^{(20)}$. Acredita-se que essa redução do controle possa ocorrer durante fonação reversa, uma vez que a manobra é atípica ao engrama pneumofônico utilizado em fala habitual e, em termos mecânicos, os próprios tecidos estão fortemente condicionados à mobilização no sentido inverso ao da fonação reversa.

A perturbação de frequência, ou Jitter, a variação da frequência fundamental em ciclos consecutivos, traduz a irregularidade da vibração da mucosa das pregas vocais, correlacionando-se com as características biomecânicas das pregas vocais e com a variação do controle neuromuscular ${ }^{(20)}, \mathrm{o}$ que converge em direção aos resultados referentes às medidas de Jitter encontrados neste estudo (Tabela 2).

A instabilidade, encontrada em um estudo ${ }^{(6)}$, foi aparente, segundo os autores, em virtude das emissões em fonação reversa de diversos sujeitos da pesquisa conterem um número significativo de trechos percentualmente similares ao som basal, qualidade vocal que sugere associação com o aumento 
da coaptação glótica. Esse aumento do fechamento glótico, associado à mobilização da mucosa, parece estar relacionado à redução, mesmo que não estatisticamente significativa, das medidas de Shimmer encontradas na presente pesquisa (Tabela 2).

A mobilização da mucosa durante a fonação reversa (a favor da gravidade) faz com que a prega vocal vibre de forma mais sincronizada durante a fonação expiratória. Assim, promove melhora da fonte do sinal laríngeo (devido à renovação do muco e à homogeneização da mucosa) e, provavelmente, também da ressonância, uma vez que o sinal glótico mais harmônico e com menos ruído é melhor propagado e modificado pelo trato ressonantal, propiciando a diminuição do ruído glótico e o aumento do número de harmônicos amplificados $^{(12,29)}$. Esse fato pode ser observado em relação às medidas de Shimmer, NHR, HNR (Tabela 2) e das sensações proprioceptivas positivas, descritas pelos sujeitos no póstécnica, como voz mais solta, melhora para falar e melhor projeção da voz (Figura 1).

As sensações vocais subjetivas relatadas pelos sujeitos da presente pesquisa, após a produção da fonação reversa, refletem o grau de consciência de suas produções vocais, além de suas concepções sobre os recursos vocais que possuem ${ }^{(9,20)}$. Neste trabalho, o relato das sensações subjetivas pós-fonação reversa, mostrou o predomínio significativo de sensações positivas e, além daquelas citadas anteriormente (voz mais solta, melhora para falar, e melhor projeção da voz), a sensação de secreção e vontade de pigarrear foram consideradas positivas (Figura 1) por refletirem a liberação do muco que reveste o epitélio das pregas vocais.

Tais sensações são coerentes com os demais resultados referentes à mobilização da mucosa, pois a presença da camada de muco sobre as pregas vocais é fundamental para a sua vibração, visto que facilita o deslize do ar transglótico pelas paredes laríngeas com o mínimo de atrito. Além disso, a mobilização da mucosa melhora a lubrificação laríngea, reduzindo a viscosidade e renovando a camada de muco ${ }^{(5,9,12,29)}$.

As alterações na camada de muco, como o aumento de sua viscosidade, reduzem as condições de adequada ocorrência do fenômeno de Bernoulli, o que faz com que as forças que atuam sobre a mucosa das pregas vocais não sejam uniformes, gerando distúrbios do ciclo vibratório e, consequentemente, alteração na qualidade acústica da voz ${ }^{(5,9,12,29)}$.

O mesmo ocorre quando a lâmina própria da mucosa das pregas vocais não é homogênea, gerando também, alterações vibratórias que se refletem em um sinal de qualidade acústica com perturbações significativas. Todos os resultados deste estudo mostram diminuição das medidas de ruído e das medidas de Shimmer consolidando as afirmações anteriores sobre a biomecânica da vibração glótica e sobre o efeito da fonação reversa, tanto sobre a fonte glótica como sobre o sinal irradiado pelos lábios, e a consequente sensação positiva da produção vocal.

No Shimmer (local, dB) e no Shimmer (apq5), a diminuição da média e a variação negativa pós-técnica mostram que houve diminuição do escape de ar e do ruído à emissão, mas com menor estabilidade desta diminuição no pós-técnica, pois o desvio-padrão aumentou, o que também reflete a efetividade dos ajustes individuais de cada sujeito ao voltar para a emissão habitual após a fonação reversa. Isto é, apenas as medidas de desvio-padrão do Shimmer aumentaram após a técnica, mostrando que os sujeitos não apresentaram comportamento homogênio ao voltar ao ajuste de fala habitual na vogal sustentada em registro modal.

Em todas as demais medidas, o desvio-padrão não se modificou de forma marcante, mostrando certa estabilidade das variações encontradas.

\section{CONCLUSÕES}

Em relação às modificações vocais produzidas pela fonação reversa, neste estudo, pôde-se concluir que, após a realização de três séries de 15 repetições em TMF, com 30 segundos de repouso passivo entre as séries, houve aumento estatisticamente significativo da $\mathrm{f}_{0} \mathrm{e}$ da frequência máxima. Além disso, percebe-se aumento das medidas de Jitter (local), de Jitter (rap), de Jitter (ppq5), e de Jitter (ddp); predomínio das sensações proprioceptivas positivas; e diminuição da frequência mínima, do Jitter (local-absoluto), das medidas de Shimmer, e das medidas de ruído, porém não com resultados estatisticamente significativos.

Pode-se inferir, por meio dos resultados da avaliação vocal acústica e de sua base anatomofisiológica que, neste estudo, a fonação reversa pareceu promover modificações mais acentuadas sobre a vibração da mucosa das pregas vocais e sobre o alongamento de prega vocal, mostrando efeito sobre a musculatura, com mudanças de $\mathrm{f}_{0}$, e sobre a mucosa, com melhora global do sinal vocal e das sensações após sua produção. Esta atividade glótica mais acentuada, porém, talvez não seja aplicável a todas as esferas da reabilitação vocal, sendo mais viável no campo de trabalho com profissionais da voz sem queixas ou alterações laríngeas como o grupo estudado. 


\begin{abstract}
Purpose: To describe the acoustic vocal modifications and the sensations occurred after the reverse phonation technique in young adult women without vocal complaints and with normal larynx. Methods: Thirty-two young adult women were submitted to otorhinolaryngologic and speech-language pathology screening to discard possible alterations that could interfere with the results of the research; had vocal samples collected before and after carrying out three series of 15 repetitions of reverse phonation, in maximum time of phonation with the habitual tone and intensity, and 30 seconds of passive rest between series. After that, they answered a questionnaire regarding their sensations. Vocal acoustic analysis was carried out using the software Praat (version 4.6.10), and the data were analyzed using descriptive statistics and the Wilcoxon test, with a 5\% level of significance. Results: Statistically significant increase of the fundamental frequency and the maximum frequency; decrease of the minimum frequency; increase of the measures of Jitter, except for the local-absolute Jitter, which diminished; decrease of Shimmer, noise-to-harmonic ratio, and harmonic-to-noise ratio measures; and predominance of positive sensations. Conclusion: Reverse phonation promoted a positive effect over the vibration of the mucosa of the vocal folds and over their stretching. This result suggests an effect over the vocal muscles, favoring changes of fundamental frequency; and over the homogenization and modification of mucus layer. Moreover, it promoted a global improvement of the vocal signal and of the sensations during its production.
\end{abstract}

Keywords: Phonation; Voice; Voice training; Voice quality; Voice disorders

\section{REFERÊNCIAS}

1. Powers WE, Holtz S, Ogura J. Contrast examination of the larynx and pharynx. Inspiratory phonation. Am J Roentgenol Radium Ther Nucl Med. 1964;92:40-2.

2. Grau SM, Robb MP, Cacace AT. Acoustic correlates of inspiratory phonation during infant cry. J Speech Hear Res. 1995;38(2):373-81.

3. Lehmann $\mathrm{QH}$. Reverse phonation: a new maneuver for examining the larynix. Radiology. 1965;84:215-22.

4. Harrisson GA, Davis PJ, Troughear RH, Winkworth AL. Inspiratory speech as a management option for spastic dysphonia. Case study. Ann Otol Rhinol Laryngol. 1992;101(5):375-82.

5. Colton RH, Casper JK. Compreendendo os problemas de voz: uma perspectiva fisiológica ao diagnóstico e ao tratamento. Porto Alegre: Artes Médicas; 1996.

6. Orlikoff RF, Baken RJ, Kraus DH. Acoustic and physiologic characteristics of inspiratory phonation. J Acoust Soc Am. 1997;102(3):1838-45.

7. Finger LS, Cielo CA. Aspectos fisiológicos e clínicos da técnica fonoterapêutica de fonação reversa. Rev Bras Otorrinolaringol. 2007;73(2):271-7.

8. Kelly CL, Fisher KV. Stroboscopic and acoustic measures of inspiratory phonation. J Voice. 1999;13(3):389-402.

9. Behlau M, Madazio G, Feijó D, Pontes PAL. Avaliação de voz. In: Behlau M, organizadora. Voz: o livro do especialista. Rio de Janeiro: Revinter; c2001. v.1, p. 85-245.

10. Pedro AO, Pinto-Neto AM, Costa-Paiva L, Osis MJ, Hardy E. Procura de serviço médico por mulheres climatéricas brasileiras. Rev Saúde Pública $=\mathrm{J}$ Public Health. 2002;36(4):484-490.

11. Santos MAO, Moura JMP, Duprat AC, Costa HO, Azevedo BB. A interferência da muda vocal nas lesões estruturais das pregas vocais. Rev Bras Otorrinolaringol. 2007;73(2):226-30.

12. Pinho SMR. Avaliação e tratamento de voz. In: Pinho SMR. Fundamentos em fonoaudiologia: tratando os distúrbios da voz. 2a ed. Rio de Janeiro: Guanabara Koogan; 2003. p. 3-40.

13. Kelchner LN, Horne J, Lee L, Klaben B, Stemple JC, Adam S, et al. Reliability of speech-language pathologist and otolaryngologist ratings of laryngeal signs of reflux in an asymptomatic population using the reflux finding score. J Voice. 2007;21(1):92-100.

14. Figueiredo LC, Gonçalves MIR, Pontes A, Pontes P. Estudo do comportamento vocal no ciclo menstrual: avaliação perceptivo-auditiva, acústica e auto-perceptiva. Rev Bras Otorrinolaringol. 2004;70(3):331-9.

15. Paes C, Vieira J, Leonel T, Cunha DA. O impacto da respiração oral no comportamento vocal. J Bras Fonoaudiol. 2005;5(23):417-23.
16. Deliyski DD, Shaw HS, Evans MK. Adverse effects of environmental noise on acoustic voice quality measurements. J Voice. 2005;19(1):15-28.

17. Deliyski DD, Evans MK, Shaw HS. Influence of data acquisition environment on accuracy of acoustic voice quality measurements. J Voice. 2005;19(2):176-86.

18. Viera MN, Rosa LLC. Avaliação acústica na prática fonoaudiológica. In: Pinho SMR, Tsuji DH, Bohadana SC. Fundamentos em laringologia e voz. Rio de Janeiro: Revinter; 2006. p. 33-51.

19. Saxon KG, Schneider CM. Vocal exercise physiology. San Diego, CA: Singular Publishing Group; 1995.

20. Barros APB, Carrara-de Angelis E. Análise acústica da voz. In: Dedivitis RA, Barros APB. Métodos de avaliação e diagnóstico de laringe e voz. São Paulo: Editora Lovise; 2002. p. 201-21.

21. Boersma P, Weenink D. Praat Manual [Internet]. Amsterdam: University of Amsterdam, Phonetic Sciences Department, 2006.[cited 2008 Dec 12]. Available from: http://www.fon.hum.uva.nl/praat/.

22. Oguz H, Tarhan E, Korkmaz M, Yilmaz U, Safak MA, Demirci M, Ozluoglu LN. Acoustic analysis findings in objective laryngopharyngeal reflux patients. J Voice. 2007;21(2):203-10.

23. Oguz H, Demirci M, Safak MA, Arslan N, Islam A, Kargin S. Effects of unilateral vocal cord paralysis on objective voice measures obtained by Praat. Eur Arch Otorhinolaryngol. 2007;264(3):257-61.

24. Dromey C, Smith ME. Vocal tremor and vibrato in the same person: acoustic and electromyographic differences. J Voice. 2008;22(5):541-5.

25. Behlau MS, Tosi O, Pontes PAL. Determinação da frequência fundamental e suas variações em altura ("jitter") e intensidade ("shimmer") para falantes do português brasileiro. Acta AWHO. 1985;4(1):5-10.

26. Araújo SA, Grellet M, Pereira JC, Rosa MO. Normatização de medidas acústicas da voz normal. Rev Bras Otorrinolaringol. 2002;68(4):540-4.

27. Felippe ACN, Grillo MHMM, Grechi TH. Normatização de medidas acústicas para vozes normais. Rev Bras Otorrinolaringol. 2006;72(5):659-64.

28. Robb MP, Chen Y, Gilbert HR, Lerman JW. Acoustic comparison of vowel articulation in normal and reverse phonation. J Speech Lang Hear Res. 2001;44(1):118-27.

29. Dedivitis RA, Barros APB. Fisiologia Laríngea. In: Dedivitis RA, Barros APB. Métodos de avaliação e diagnóstico de laringe e voz. São Paulo: Editora Lovise; 2002. p. 38-52.

30. Sulica L, Behrman A, Roark R. The inspiratory maneuver: a simple method to assess the superficial lamina propria during endoscopy. J Voice. 2005;19(3):481-4. 